\title{
A RIGIDITY RESULT FOR KÄHLERIAN MANIFOLDS ENDOWED WITH CLOSED CONFORMAL VECTOR FIELDS
}

\author{
ANTONIO CAMINHA
}

\begin{abstract}
We show that if a connected compact Kählerian surface $M$ with nonpositive Gaussian curvature is endowed with a closed conformal vector field $\xi$ whose singular points are isolated, then $M$ is isometric to a flat torus and $\xi$ is parallel. We also consider the case of a connected complete Kählerian manifod $M$ of complex dimension $n>1$ and endowed with a nontrivial closed conformal vector field $\xi$. In this case, it is well known that the singularities of $\xi$ are automatically isolated and the nontrivial leaves of the distribution generated by $\xi$ and $J \xi$ are totally geodesic in $M$. Assuming that one such leaf is compact, has torsion normal holonomy group and that the holomorphic sectional curvature of $M$ along it is nonpositive, we show that $\xi$ is parallel and $M$ is foliated by a family of totally geodesic isometric tori and also by a family of totally geodesic isometric complete Kählerian manifolds of complex dimension $n-1$. In particular, the universal covering of $M$ is isometric to a Riemannian product having $\mathbb{R}^{2}$ as a factor. We also comment on a generic class of compact complex symmetric spaces not possessing nontrivial closed conformal vector fields, thus showing that we cannot get rid of the hypothesis of nonpositivity of the holomorphic sectional curvature in the direction of $\xi$.
\end{abstract}

\section{INTRODUCTION}

A conformal vector field $\xi$ on a semi-Riemannian manifold $M$ is closed if its metrically dual 1 -form is closed. In this case, if $\nabla$ stands for the Levi-Civita connection of $M$, one has $\nabla_{X} \xi=\psi X$ for some smooth function $\psi$ on $M$ (the conformal factor of $\xi$ ) and every $X \in \mathfrak{X}(M)$.

The geometry of Riemannian submanifolds of Lorentzian and Riemannian manifolds in which either the submanifold or the ambient space is endowed with a closed conformal vector field has been the object of intense research in recent years (see, for instance, [1, 3, 6, 7] and the references therein). The presence of such a (nontrivial) vector field also imposes strong restrictions on the structure of the ambient manifold $M$ itself. For example, it is a well known fact (cf. [4] and [7]) that, in a neighborhood of each nonsingular point, $M$ is isometric to a warped product structure.

2010 Mathematics Subject Classification. 53B35, 53C55, 53C35.

Key words and phrases. Kählerian metrics; Closed conformal vector fields; Geometric foliations; Symmetric spaces. 
In what concerns Kählerian manifolds, a canonical class of examples possessing closed conformal vector fields is that of the warped products $M^{n}=I \times_{t} N^{2 n-1}$ ( $n$ standing for the complex dimension of $M$ and $t$ for the $I$-variable), where $N$ is a $(2 n-1)$-dimensional Sasakian manifold; in this case, $\xi=t \partial_{t}$ is closed conformal and $\psi=1$ is its conformal factor. Another one is that of the Riemannian products $M^{n}=N^{n-1} \times \mathbb{T}$, where $N$ is a Kählerian manifold of complex dimension $n-1$ and $\mathbb{T}$ is a flat torus of complex dimension 1 ; in this case, the conformal vector field $\xi$ is the lift, to $M$, of a parallel vector field in $\mathbb{T}$, and $\psi=0$ is its conformal factor. In both of these classes of examples, the holomorphic sectional curvature of $M$ in the direction of $\xi$ vanishes identically and, if $J$ stands for the quasi-complex structure of $M$, then the leaves of the distribution generated by $\xi$ and $J \xi$ are totally geodesic in $M$. However, in the first class such leaves are noncompact, whereas in the second class they are compact.

On the other hand, if $\left(M^{n}, J, g\right)$ is a compact complex symmetric space of complex dimension $n$, positive scalar curvature, vanishing first De Rham cohomology group and not isometric to $\mathbb{S}^{2 n}$, then [8, Theorem 1] implies that $M$ does not possess a nontrivial closed conformal vector field. A particular instance of this situation is that of $\mathbb{C P}^{n}$ endowed with the Fubini-Study metric. Therefore, covering space theory shows that if $\left(M^{n}, J, g\right)$ is a compact connected Kählerian manifold of positive constant holomorphic sectional curvature ( $n$ being its complex dimension), then $M$ does not possess a nontrivial closed conformal vector field.

The purpose of this paper is to show that, under a reasonable set of conditions on the closed conformal vector field $\xi$, the second class of examples presented in the third paragraph is essentially the only one. More precisely, we first show that if a connected compact Riemann surface $M$, endowed with a Kählerian metric of nonpositive Gaussian curvature, possesses a closed conformal vector field $\xi$ whose singular points are isolated, then $M$ is isometric to a flat torus and $\xi$ is parallel. We then consider the case of a connected complete Kählerian manifod $M^{n}$, of complex dimension $n>1$ and endowed with a nontrivial closed conformal vector field $\xi$. In this case, it is a well known fact (cf. [7]) that the singularities of $\xi$ are automatically isolated and the nontrivial leaves of the distribution generated by $\xi$ and $J \xi$ are totally geodesic in $M$. Assuming that one such leaf is compact, has torsion normal holonomy group and that the holomorphic sectional curvature of $M$ along it is nonpositive, we show that $\xi$ is parallel and $M$ is foliated by a family of totally geodesic isometric tori, and also by a family of totally geodesic, isometric complete Kählerian manifolds of complex dimension $n-1$. In particular, the universal covering of $M$ is isometric to a Riemannian product having $\mathbb{R}^{2}$ as a factor.

Our approach is based on a certain kind of deformation of the original Kählerian metric of $M$, which is interesting in itself and generalizes the way the metric of $\mathbb{C}^{n}$ deforms into that of $\mathbb{C} \mathbb{H}^{n}$. 


\section{DeForming KäHLERIAN METRICS}

In the sequel, we let $\left(M^{n}, J, g\right)$ be an $n$-dimensional Hermitian manifold, where $n$ stands for its complex dimension (hence, $M$ has real dimension $2 n$ ). We also let $\omega \in \Omega^{2}(M)$ denote the corresponding Kählerian form, so that $\omega(X, Y)=\langle J X, Y\rangle$ for all $X, Y \in \mathfrak{X}(M)$. It is a standard fact that $M$ is a Kählerian manifold if and only if $J$ is parallel with respect to the Levi-Civita connection $\nabla$ of $g$.

Whenever convenient, we write $g=\langle\cdot, \cdot\rangle$ and let $|\cdot|$ denote the corresponding norm. Also, for $X \in \mathfrak{X}(M)$, we let $\theta_{X}$ denote the 1 -form metrically dual to $X$, so that $\theta_{X}(Y)=\langle X, Y\rangle$ for $Y \in \mathfrak{X}(M)$; we also let $\theta_{X}^{2}$ denote the symmetrization of $\theta_{X} \otimes \theta_{X}$.

We recall that a conformal vector field $\xi$ on $(M, g, \nabla)$ is said to be closed conformal if $\theta_{\xi}$ is a closed 1 -form. These conditions are readily seen to be equivalent to the existence of a smooth function $\psi$ on $M$ (called the conformal factor of $\xi$ ) such that $\nabla_{X} \xi=\psi X$ for all $X \in \mathfrak{X}(M)$.

If $(M, J, g, \nabla)$ is endowed with a nontrivial closed conformal vector field, the following result presents a simple way to construct, out of $g$, a new Kählerian metric on $(M, J)$.

Proposition 2.1. Let $(M, J, g=\langle\cdot, \cdot\rangle)$ be a Kählerian manifold with Levi-Civita connection $\nabla$, and $\xi \in \mathfrak{X}(M)$ be a nontrivial closed conformal vector field on $M$. If $|\xi|^{2}<c$ on $M$ for some positive constant $c$ and $\mu=\left(c-|\xi|^{2}\right)^{-1}$, then the covariant symmetric 2-tensor field

$$
\tilde{g}=\mu g+\mu^{2}\left(\theta_{\xi}^{2}+\theta_{J \xi}^{2}\right)
$$

defines another Kählerian metric on $(M, J)$.

Proof. We briefly sketch the (simple) proof. The 2-tensor $\tilde{g}$ is clearly positive definite, and thus defines a Riemannian metric on $M$. On the other hand, for $X, Y \in \mathfrak{X}(M)$, the Hermitian character of $\langle\cdot, \cdot\rangle$ with respect to $J$ readily implies that of $\tilde{g}$ (also with respect to $J$ ).

Next, if $\omega$ and $\tilde{\omega}$ stand for the Kählerian forms of $(M, J, g)$ and $(M, J, \tilde{g})$, respectively, then another straightforward computation gives $\tilde{\omega}=\mu \omega+\mu^{2} \theta_{\xi} \wedge \theta_{J \xi}$, and hence

$$
d \tilde{\omega}=d \mu \wedge \omega+2 \mu d \mu \wedge \theta_{\xi} \wedge \theta_{J \xi}-\mu^{2} \theta_{\xi} \wedge d \theta_{J \xi}
$$

Letting $\psi$ be the conformal factor of $\xi$ and $X \in \mathfrak{X}(M)$, one also computes $d \mu=$ $2 \psi \mu^{2} \theta_{\xi}$ and $d \theta_{J \xi}=2 \psi \omega$. Therefore,

$$
d \tilde{\omega}=2 \psi \mu^{2} \theta_{\xi} \wedge \omega+4 \psi \mu^{3} \theta_{\xi} \wedge \theta_{\xi} \wedge \theta_{J \xi}-\mu^{2} \theta_{\xi} \wedge(2 \psi \omega)=0 .
$$

Our next result gives a set of conditions under which $(M, J, \tilde{g})$ is a complete Riemannian manifold.

Lemma 2.2. Under the hypotheses of Proposition 2.1, assume that the conformal factor $\psi$ of $\xi$ is bounded and does not vanish outside a compact subset of $M$. If $|\xi|^{2}: M \rightarrow[0,+\infty)$ is proper and such that $\sup _{M}|\xi|^{2}=c$, then $(M, \tilde{g})$ is complete.

Proof. Let $\tilde{\ell}(\cdot)$ denote length with respect to $\tilde{g}$. Standard Riemannian geometry assures that it suffices to show that if a smooth curve $\gamma:[0,+\infty) \rightarrow M$ escapes 
from all compact subsets of $M$, then $\tilde{\ell}(\gamma)=+\infty$. To this end, we start by observing that

$$
\tilde{g}(v, v)=\mu g(v, v)+\mu^{2}\left(\langle\xi, v\rangle^{2}+\langle J \xi, v\rangle^{2}\right) \geq(\mu\langle\xi, v\rangle)^{2} .
$$

Now, choose $K \subset M$ to be a compact set such that $\psi \neq 0$ on $K^{c}$, and $t_{0}>0$ such that $\gamma(t) \notin K$ for $t>t_{0}$. Letting $\sup _{M}|\psi|=\alpha<+\infty$, we estimate

$$
\begin{aligned}
\tilde{\ell}\left(\gamma_{\mid[0, t]}\right) & \geq \int_{t_{0}}^{t} \tilde{g}\left(\gamma^{\prime}(s), \gamma^{\prime}(s)\right)^{1 / 2} d s \geq \int_{t_{0}}^{t}\left|\mu(\gamma(s))\left\langle\xi(\gamma(s)), \gamma^{\prime}(s)\right\rangle\right| d s \\
& =\int_{t_{0}}^{t} \frac{1}{|\psi(\gamma(s))|} \cdot \frac{1}{c-|\xi(\gamma(s))|^{2}}\left|\left\langle\xi(\gamma(s)), \nabla_{\gamma^{\prime}(s)} \xi\right\rangle\right| d s \\
& \geq\left.\frac{1}{2 \alpha}\left|\int_{t_{0}}^{t} \frac{1}{c-|\xi(\gamma(s))|^{2}} \frac{d}{d s}\right| \xi(\gamma(s))\right|^{2} d s \mid \\
& =\frac{1}{2 \alpha}\left|\log \left(c-\left|\xi\left(\gamma\left(t_{0}\right)\right)\right|^{2}\right)-\log \left(c-|\xi(\gamma(t))|^{2}\right)\right| .
\end{aligned}
$$

Let $\epsilon>0$ be given. Since $|\xi|^{2}$ is proper, $|\xi|^{2}<c$ and $\sup _{M}|\xi|^{2}=c$, there exists a compact subset $L_{\epsilon}$ of $M$ such that $|\xi|^{2}>c-\epsilon$ in $L_{\epsilon}^{c}$. Since $\gamma$ is divergent, there exists $t_{\epsilon}>t_{0}$ such that $\gamma(t) \in L_{\epsilon}^{c}$ for $t>t_{\epsilon}$. Hence, for $t>t_{0}, t_{\epsilon}$, the above computations give

$$
\tilde{\ell}\left(\gamma_{\mid[0, t]}\right) \geq \frac{1}{2 \alpha}\left(\log \left(c-\left|\xi\left(\gamma\left(t_{0}\right)\right)\right|^{2}\right)-\log \epsilon\right),
$$

so that $\tilde{\ell}(\gamma)=\lim _{t \rightarrow+\infty} \tilde{\ell}\left(\gamma_{\mid[0, t]}\right)=+\infty$.

Remark 2.3. The previous result continues to hold if we assume that (in the notations of the proof) $\psi^{-1}(0) \cap K^{c}$ is a set of isolated points. It suffices to split the trace of $\gamma$ into pieces along each of which $\psi \neq 0$, estimate the length of each such piece as we did above and add the results.

Example 2.4. In the complex Euclidean $n$-space $\mathbb{C}^{n}$, let $J$ be the standard quasicomplex structure, $g=\langle\cdot, \cdot\rangle$ the standard metric and $\mathbb{B}^{n}=\left\{z \in \mathbb{C}^{n} ;|z|<1\right\}$. Since the vector field $\xi(p)=p$ is closed and conformal, the previous construction endows $\mathbb{B}^{n}$ with a Kählerian metric $\tilde{g}$, such that

$$
\tilde{g}=\frac{1}{1-|\xi|^{2}}\langle\cdot, \cdot\rangle+\frac{1}{\left(1-|\xi|^{2}\right)^{2}}\left(\theta_{\xi}^{2}+\theta_{J \xi}^{2}\right) .
$$

An immediate application of the previous result establishes the completeness of $\left(\mathbb{B}^{n}, \tilde{g}\right)$. Therefore, the formula of Lemma 2.7 for the holomorphic sectional curvature of $\left(\mathbb{B}^{n}, J, \tilde{g}\right)$, together with the Hawley-Igusa theorem, shows that $\left(\mathbb{B}^{n}, J, \tilde{g}\right)$ is nothing but the complex hyperbolic space $\mathbb{C H}^{n}$.

Example 2.5. Let $\left(N^{n-1}, J_{N}, g_{N}\right)$ be a Kählerian manifold (of complex dimension $n-1)$ and $\mathbb{T}$ be a flat torus with its standard quasi-complex structure. If $M^{n}=$ $N \times \mathbb{T}$ is endowed with the product quasi-complex structure and the product metric, then $M^{n}$ is a Kählerian manifold. Moreover, if $\mathbb{T}$ is the quotient of the lattice $\mathcal{L}$ in $\mathbb{R}^{2}$ and $Z$ stands for the canonical vector field along one of the directions of the 
lattice, then $Z$ induces a nontrivial parallel smooth vector field on $\mathbb{T}$, which can be lifted to a corresponding one on $M$.

Henceforth, we let $\left(M^{n}, J, g\right)$ be a Kählerian manifold, $\xi \in \mathfrak{X}(M)$ be a nontrivial closed conformal vector field with conformal factor $\psi$ and such that $|\xi|^{2}<c$, and $\tilde{g}$ be the Kählerian metric on $(M, J)$ given as in Proposition 2.1. We want to relate the holomorphic sectional curvatures of $(M, J, g)$ and $(M, J, \tilde{g})$, and to this end we start by relating their Levi-Civita connections.

Lemma 2.6. If $\nabla$ and $\tilde{\nabla}$ stand for the Levi-Civita connections of $g$ and $\tilde{g}$, respectively, then

$$
\tilde{\nabla}_{X} Y=\nabla_{X} Y+\psi \mu(\langle\xi, X\rangle Y+\langle\xi, Y\rangle X+\langle J \xi, X\rangle J Y+\langle J \xi, Y\rangle J X) .
$$

Proof. The proof is a somewhat lengthy, though straightforward computation. On the one hand, for $X, Y, Z \in \mathfrak{X}(M)$ we have

$$
2 \tilde{g}\left(\tilde{\nabla}_{X} Y, Z\right)=2 \mu\left\langle\tilde{\nabla}_{X} Y+\mu\left\langle\tilde{\nabla}_{X} Y, \xi\right\rangle \xi+\mu\left\langle\tilde{\nabla}_{X} Y, J \xi\right\rangle J \xi, Z\right\rangle .
$$

On the other, Koszul's formula gives

$$
\begin{aligned}
2 \tilde{g}\left(\tilde{\nabla}_{X} Y, Z\right)= & X(\tilde{g}(Y, Z))+Y(\tilde{g}(Z, X))-Z(\tilde{g}(X, Y)) \\
& -\tilde{g}(X,[Y, Z])+\tilde{g}(Y,[Z, X])+\tilde{g}(Z,[X, Y]) .
\end{aligned}
$$

By computing each summand at the right hand side of the last expression above we obtain, after some cancellations,

$$
\begin{aligned}
2 \tilde{g}\left(\tilde{\nabla}_{X} Y, Z\right)= & 2 \mu\left\langle\nabla_{X} Y+\psi \mu(\langle\xi, X\rangle Y+\langle\xi, Y\rangle X)\right. \\
& +2 \psi \mu^{2}(\langle\xi, X\rangle\langle\xi, Y\rangle \xi+\langle\xi, X\rangle\langle J \xi, Y\rangle J \xi) \\
& +2 \psi \mu^{2}(\langle J \xi, X\rangle\langle\xi, Y\rangle J \xi-\langle J \xi, X\rangle\langle J \xi, Y\rangle \xi) \\
& +\mu\left(\left\langle\nabla_{X} Y, \xi\right\rangle \xi+\left\langle\nabla_{X} Y, J \xi\right\rangle J \xi\right) \\
& +\mu(\psi\langle J \xi, Y\rangle J X+\psi\langle J \xi, X\rangle J Y), Z\rangle .
\end{aligned}
$$

Setting $W=\tilde{\nabla}_{X} Y-\nabla_{X} Y$ and comparing the two expressions for $2 \tilde{g}\left(\tilde{\nabla}_{X} Y, Z\right)$, we arrive at

$$
W+\mu\langle W, \xi\rangle \xi+\mu\langle W, J \xi\rangle J \xi=\mu F(X, Y),
$$

where

$$
\begin{aligned}
F(X, Y)= & \psi(\langle\xi, X\rangle Y+\langle\xi, Y\rangle X+\langle J \xi, Y\rangle J X+\langle J \xi, X\rangle J Y) \\
& +2 \psi \mu(\langle\xi, X\rangle\langle\xi, Y\rangle-\langle J \xi, X\rangle\langle J \xi, Y\rangle) \xi \\
& +2 \psi \mu(\langle\xi, X\rangle\langle J \xi, Y\rangle+\langle J \xi, X\rangle\langle\xi, Y\rangle) J \xi .
\end{aligned}
$$

Taking the inner product of (2.3) with $\xi$ and $J \xi$, respectively, and recalling that $1+\mu\langle\xi, \xi\rangle=c \mu$, we successively get $\langle W, \xi\rangle=c^{-1}\langle F(X, Y), \xi\rangle$ and $\langle W, J \xi\rangle=$ $c^{-1}\langle F(X, Y), J \xi\rangle$, whence

$$
W=\mu\left(F(X, Y)-c^{-1}\langle F(X, Y), \xi\rangle \xi-c^{-1}\langle F(X, Y), J \xi\rangle J \xi\right) .
$$

However, $\langle J \xi, \xi\rangle=0$ gives $\langle F(X, Y), \xi\rangle=2 \psi \mu c(\langle\xi, X\rangle\langle\xi, Y\rangle-\langle J \xi, X\rangle\langle J \xi, Y\rangle)$ and $\langle F(X, Y), J \xi\rangle J=2 \psi \mu c(\langle\xi, X\rangle\langle J \xi, Y\rangle+\langle\xi, Y\rangle\langle J \xi, X\rangle)$. Substituting these formulas in the right hand side of the expression of $W$, we arrive at (2.2). 
Before we can proceed to relate the holomorphic sectional curvatures of $g=\langle\cdot, \cdot\rangle$ and $\tilde{g}$, we need a few more preliminaries. Firstly, the closed conformal condition on $\xi \in \mathfrak{X}\left(M^{n}\right)$ (recall that $n$ stands for the complex dimension of $M$ ) readily gives

$$
\psi=\frac{1}{2 n} \operatorname{div} \xi
$$

and Lemma 1 of [7] shows that

$$
|\xi|^{2} \nabla(\operatorname{div} \xi)=-2 n \operatorname{Ric}(\xi) \xi
$$

where $\nabla(\operatorname{div} \xi)$ stands for the gradient of the divergence of $\xi$ with respect to $g$ and $\operatorname{Ric}(\xi)$ for the normalized Ricci curvature of $(M, g)$ in the direction of $\xi$. In particular, at each point where $\xi \neq 0$, we get

$$
\nabla \psi=-\operatorname{Ric}(\hat{\xi}) \xi
$$

where $\hat{\xi}=\frac{\xi}{|\xi|}$.

Lemma 2.7. Let $\left(M^{n}, J, g\right)$ be a Kählerian manifold, $\xi \in \mathfrak{X}(M)$ be a closed conformal vector field with isolated zeros and such that $|\xi|^{2}<c$ on $M, \psi$ be the conformal factor of $\xi$, and $\tilde{g}$ be the Kählerian metric on $(M, J)$ given as in Proposition 2.1. For $X \in T_{p} M$ unitary with respect to $g$, let $K(X)$ and $\tilde{K}(X)$ denote the holomorphic sectional curvatures of $(M, J, g)$ and $(M, J, \tilde{g})$ with respect to $X$, respectively. Then,

$$
\begin{aligned}
\tilde{K}(X)= & \frac{1}{\tilde{g}(X, X)^{2}}\left\{\mu K(X)+\mu^{2} \operatorname{Ric}(\hat{\xi})\left(\langle X, \xi\rangle^{2}+\langle X, J \xi\rangle^{2}\right)\right\} \\
& +\frac{1}{\tilde{g}(X, X)} \cdot 2 \mu \operatorname{Ric}(\hat{\xi})\left(\langle X, \xi\rangle^{2}+\langle X, J \xi\rangle^{2}\right)-4 \psi^{2},
\end{aligned}
$$

with $\operatorname{Ric}(\hat{\xi})$ being taken as 0 if $\xi(p)=0$.

Proof. We first perform the computations at a point $p \in M$ such that $\xi(p) \neq 0$. Letting $\tilde{R}$ denote the curvature tensor of $(M, \tilde{g})$, we have

$$
\begin{aligned}
& \tilde{K}(X)= \frac{\tilde{g}(\tilde{R}(X, J X) J X, X)}{\tilde{g}(X, X) \tilde{g}(J X, J X)-\tilde{g}(X, J X)^{2}} \\
&=\frac{1}{\tilde{g}(X, X)^{2}}\left\{X\left(\tilde{g}\left(\tilde{\nabla}_{J X} J X, X\right)\right)-\tilde{g}\left(\tilde{\nabla}_{J X} J X, \tilde{\nabla}_{X} X\right)-J X\left(\tilde{g}\left(\tilde{\nabla}_{X} J X, X\right)\right)\right. \\
&\left.\quad+\tilde{g}\left(\tilde{\nabla}_{X} J X, \tilde{\nabla}_{J X} X\right)-\tilde{g}\left(\tilde{\nabla}_{[X, J X]} J X, X\right)\right\} .
\end{aligned}
$$

Extend $X$ to a neighborhood of $p$, with $\left(\nabla_{v} X\right)(p)=0$ and $\left(\nabla_{v} J X\right)(p)=0$ for all $v \in T_{p} M$ (with $e_{1}(p)=X_{p}$, the parallelism of $J$ allows us to take a Hermitian geodesic frame $\left(e_{1}, J e_{1}, \ldots, e_{n}, J e_{n}\right)$ around $\left.p\right)$; hence, $[X, J X]=0$ at $p$. Setting $\alpha=2 \psi \mu\langle X, \xi\rangle$ and $\beta=2 \psi \mu\langle X, J \xi\rangle$, relation (2.2) gives, at the point $p$ and after 
some straightforward computations,

$$
\begin{aligned}
\tilde{K}(X)= & \frac{1}{\tilde{g}(X, X)^{2}}\{\underbrace{X\left(\tilde{g}\left(\nabla_{J X} J X, X\right)\right)-J X\left(\tilde{g}\left(\nabla_{X} J X, X\right)\right)}_{I}\} \\
& +\frac{1}{\tilde{g}(X, X)}\{\underbrace{-X(\alpha)+2\left(\alpha^{2}+\beta^{2}\right)+J X(\beta)}_{I I}\} \\
& +\frac{1}{\tilde{g}(X, X)^{2}}\{\underbrace{-\alpha X(\tilde{g}(X, X))+\beta J X(\tilde{g}(X, X))}_{I I I}\} .
\end{aligned}
$$

Computing each of $I, I I$ and $I I I$ at $p$, we get

$$
\begin{aligned}
I=\mu K(X) & +\mu^{2}\langle R(X, J X) J X, \xi\rangle\langle X, \xi\rangle+\mu^{2}\langle R(X, J X) J X, J \xi\rangle\langle X, J \xi\rangle, \\
I I= & 2 \mu \operatorname{Ric}(\hat{\xi})\langle X, \xi\rangle^{2}+2 \mu \operatorname{Ric}(\hat{\xi})\langle X, J \xi\rangle^{2} \\
& +4 \psi^{2}\left(\mu+\mu^{2}\left(\langle X, \xi\rangle^{2}+\langle X, J \xi\rangle^{2}\right)\right)-8 \psi^{2} \mu \\
= & 2 \mu \operatorname{Ric}(\hat{\xi})\left(\langle X, \xi\rangle^{2}+\langle X, J \xi\rangle^{2}\right)+4 \psi^{2} \tilde{g}(X, X)-8 \psi^{2} \mu,
\end{aligned}
$$

and

$$
\begin{aligned}
I I I= & -2 \psi \mu\langle X, \xi\rangle\left\{4 \psi \mu\langle X, \xi\rangle \tilde{g}(X, X)+2 \psi \mu^{2}\langle X, \xi\rangle-2 \psi \mu^{2}\langle X, \xi\rangle\right\} \\
& +2 \psi \mu\langle X, J \xi\rangle\left\{4 \psi \mu\langle J X, \xi\rangle \tilde{g}(X, X)-2 \psi \mu^{2}\langle X, J \xi\rangle-2 \psi \mu^{2}\langle J X, \xi\rangle\right\} \\
= & -8 \psi^{2} \mu^{2}\left(\langle X, \xi\rangle^{2}+\langle X, J \xi\rangle^{2}\right) \tilde{g}(X, X) .
\end{aligned}
$$

Substituting these expressions in (2.6), we obtain

$$
\begin{aligned}
\tilde{K}(X)= & \frac{1}{\tilde{g}(X, X)^{2}}\left\{\mu K(X)+\mu^{2}\langle R(X, J X) J X, \xi\rangle\langle X, \xi\rangle\right. \\
& \left.+\mu^{2}\langle R(X, J X) J X, J \xi\rangle\langle X, J \xi\rangle\right\} \\
& +\frac{1}{\tilde{g}(X, X)} \cdot 2 \mu \operatorname{Ric}(\hat{\xi})\left(\langle X, \xi\rangle^{2}+\langle X, J \xi\rangle^{2}\right)-4 \psi^{2} .
\end{aligned}
$$

Now, by invoking Lemma 1 of [7] once more, we obtain

$$
\begin{aligned}
\langle R(X, J X) J X, \xi\rangle & =-\operatorname{Ric}(\hat{\xi})(\langle J X, \xi\rangle\langle X, J X\rangle-\langle X, \xi\rangle\langle J X, J X\rangle) \\
& =\operatorname{Ric}(\hat{\xi})\langle X, \xi\rangle
\end{aligned}
$$

and, similarly, $\langle R(X, J X) J X, J \xi\rangle=\operatorname{Ric}(\hat{\xi})\langle X, J \xi\rangle$. Substituting these formulas in the last expression above for $\tilde{K}(X)$, we finally arrive at the formula displayed in the statement of the theorem.

If $\xi(p)=0$, take a sequence $\left(p_{j}\right)_{j \geq 1}$ in $M$, converging to $p$ and such that $\xi\left(p_{j}\right) \neq 0$ (such a sequence does exist, for we are assuming that the zeros of $\xi$ are isolated). Compute (2.5) at $p_{j}$ and let $j \rightarrow+\infty$. Since $\operatorname{Ric}\left(\hat{\xi}\left(p_{j}\right)\right)\left(\langle X, \xi\rangle_{p_{j}}^{2}+\langle X, J \xi\rangle_{p_{j}}^{2}\right) \stackrel{j}{\longrightarrow} 0$, that relation is still valid at $p$, provided we interpret $\operatorname{Ric}(\hat{\xi})$ as equal to 0 at $p$.

The corollary below extends, to a general deformation $g \mapsto \tilde{g}$ as above, the phenomenon of holomorphic sectional curvature decay that takes place when we pass from $\mathbb{C}^{n}$ to $\mathbb{C} \mathbb{H}^{n}$. 
Corollary 2.8. Let $(M, J, g)$ be a Kählerian manifold, $\xi \in \mathfrak{X}(M)$ be a closed conformal vector field with isolated zeros and such that $|\xi|^{2}<c$ on $M$, and $\tilde{g}$ be the Kählerian metric on $(M, J)$ given as in Proposition 2.1. For $X \in T_{p} M$ unitary with respect to $g$ and such that $K(X) \geq 0$, we have:

(a) If $X \perp \xi$, J $\xi$, then $\tilde{K}(X) \leq c K(X)-4 \psi^{2}$.

(b) For a general $X$, and if $M$ has nonnegative Ricci curvature, then

$$
\tilde{K}(X) \leq c K(X)+2 c \operatorname{Ric}(\hat{\xi})-4 \psi^{2},
$$

with $\operatorname{Ric}(\hat{\xi})$ being taken as 0 if $\xi(p)=0$.

Proof. If $X \perp \xi, J \xi$, then $\tilde{g}(X, X)=\mu$. Therefore, our previous result gives

$$
\tilde{K}(X)=\left(c-|\xi|^{2}\right) K(X)-4 \psi^{2} \leq c K(X)-4 \psi^{2} .
$$

For a general $X \in T_{p} M$ unitary, let $A=\langle X, \xi\rangle^{2}+\langle X, J \xi\rangle^{2}$ and write

$$
\tilde{K}(X)=\frac{\mu}{\tilde{g}(X, X)^{2}} \cdot K(X)+\frac{\left(\mu^{2}+2 \mu \tilde{g}(X, X)\right) A}{\tilde{g}(X, X)^{2}} \cdot \operatorname{Ric}(\hat{\xi})-4 \psi^{2},
$$

For the first summand, note that

$$
1+\mu A=\frac{c-|\xi|^{2}+\langle X, \xi\rangle^{2}+\langle J X, \xi\rangle^{2}}{c-|\xi|^{2}} \geq \frac{c-|\xi|^{2}}{c-|\xi|^{2}}=1
$$

hence,

$$
\frac{\mu}{\tilde{g}(X, X)^{2}}=\frac{1}{\mu(1+\mu A)^{2}} \leq \frac{1}{\mu} \leq c .
$$

For the second summand, substituting $\tilde{g}(X, X)=\mu+\mu^{2} A$ we get

$$
\frac{\left(\mu^{2}+2 \mu \tilde{g}(X, X)\right) A}{\tilde{g}(X, X)^{2}}=\frac{(3+2 \mu A) A}{(1+\mu A)^{2}}=\frac{1}{\mu} \cdot \frac{3 y+2 y^{2}}{1+2 y+y^{2}},
$$

where $y=\mu A$. It now suffices to observe that $\frac{1}{\mu} \leq c$ and (since $y \geq 0$ )

$$
\frac{3 y+2 y^{2}}{1+2 y+y^{2}}=2-\frac{1}{y+1}-\frac{1}{(y+1)^{2}}<2 \text {. }
$$

\section{TWO RIGIDITY RESULTS FOR KÄHLERIAN MANIFOLDS}

We now use the metric deformation discussed in the previous section to study the structure of a connected complete Kählerian manifold endowed with a nontrivial closed conformal vector field. We start by looking at the compact case, for which we need the following result.

Lemma 3.1. Let $\left(M^{1}, g, J\right)$ be a compact Kählerian surface endowed with a closed conformal vector field $\xi \in \mathfrak{X}(M)$, with isolated zeros and conformal factor $\psi$. Let $c>0$ be such that $c>\max _{M}|\xi|^{2}$ and $\tilde{g}$ be the metric on $M$ defined as in (2.1). If $d M_{g}$ and $d M_{\tilde{g}}$ stand for the volume elements of $M$ with respect to $g$ and $\tilde{g}$, respectively, then $d M=c \mu^{2} d M_{g}$. 
Proof. Since the singular points of $\xi$ are isolated by hypothesis, it suffices to deduce the stated relation at the points where $\xi \neq 0$. At such a point $p$, if $e_{1}=\frac{\xi}{\tilde{g}(\xi, \xi)^{1 / 2}}=$ $\frac{\xi}{\sqrt{c} \mu|\xi|}$ and $e_{2}=\frac{J \xi}{\tilde{g}(\xi, \xi)^{1 / 2}}=\frac{J \xi}{\sqrt{c} \mu|\xi|}$, then $\left\{e_{1}, e_{2}\right\}$ is a positive orthonormal basis of $T_{p} M$ with respect to $\tilde{g}$. Hence, if $\left\{\tilde{\theta}_{1}, \tilde{\theta}_{2}\right\}$ is the metrically dual basis, then $d_{\tilde{g}} M=\tilde{\theta}_{1} \wedge \tilde{\theta}_{2}$ at $p$. For a vector $v \in T_{p} M$, a direct computation gives

$$
\tilde{\theta}_{1}(v)=\frac{1}{\sqrt{c} \mu|\xi|} \tilde{g}(v, \xi)=\frac{1}{\sqrt{c} \mu|\xi|}\left(\mu+\mu^{2}|\xi|^{2}\right) \theta_{\xi}(v)=\sqrt{c} \mu \theta_{\hat{\xi}}(v),
$$

so that $\tilde{\theta}_{1}=\sqrt{c} \mu \theta_{\hat{\xi}}$. Likewise, $\tilde{\theta}_{2}=\sqrt{c} \mu \theta_{J \hat{\xi}}$. However, since $\{\hat{\xi}, J \hat{\xi}\}$ is a positive orthonormal basis for $T_{p} M$ with respect to $g$, we have

$$
d M_{\tilde{g}}=\tilde{\theta}_{1} \wedge \tilde{\theta}_{2}=c \mu^{2} \theta_{\hat{\xi}} \wedge \theta_{J \hat{\xi}}=c \mu^{2} d M_{g}
$$

We have finally arrived at our first main result.

Theorem 3.2. Let $\left(M^{1}, g, J\right)$ be a connected, compact Kählerian surface with Gaussian curvature $K \leq 0$. If $M$ possesses a closed conformal vector field $\xi \in$ $\mathfrak{X}(M)$ whose zeros are all isolated, then $K \equiv 0, \xi$ is parallel, and $M$ is isometric to a flat torus.

Proof. As before, let $\psi$ be the conformal factor of $\xi$, choose a real number $c>0$ such that $c>\max _{M}|\xi|^{2}$, and let $\tilde{g}$ be defined as in (2.1).

Since $\xi^{-1}(0)$ is a set of isolated points and $M$ is compact, we conclude that $\xi^{-1}(0)$ is finite. Therefore, if $\tilde{K}$ stands for the Gaussian curvature of $(M, \tilde{g})$, then, at every point of $M \backslash \xi^{-1}(0)$ and in the notations of Lemma 2.7, we have $K=K(\hat{\xi})$ and $\tilde{K}=\tilde{K}(\hat{\xi})$. That result also furnishes

$$
\tilde{K}=\frac{1}{\tilde{g}(\hat{\xi}, \hat{\xi})^{2}}\left\{\mu K+\mu^{2} K\left(\langle\hat{\xi}, \xi\rangle^{2}+\langle\hat{\xi}, J \xi\rangle^{2}\right)\right\}+\frac{1}{\tilde{g}(\hat{\xi}, \hat{\xi})} \cdot 2 \mu K\left(\langle\hat{\xi}, \xi\rangle^{2}+\langle\hat{\xi}, J \xi\rangle^{2}\right)-4 \psi^{2}
$$

Since $\langle\hat{\xi}, J \xi\rangle=0$ and $\tilde{g}(\hat{\xi}, \hat{\xi})=c \mu^{2}$, we obtain, after some simple algebra,

$$
\tilde{K}=\left(\frac{c+|\xi|^{2}}{c \mu}\right) K-4 \psi^{2}
$$

By continuity, this last formula relating $K$ and $\tilde{K}$ holds in all of $M$.

We now apply Gauss-Bonnet theorem twice, with the aid of Lemma 3.1

$$
\begin{aligned}
2 \pi \mathcal{X}(M) & =\int_{M} \tilde{K} d M_{\tilde{g}}=\int_{M}\left(\frac{c+|\xi|^{2}}{c-|\xi|^{2}}\right) K d M_{g}-4 c \int_{M} \psi^{2} \mu^{2} d M_{g} \\
& \leq \int_{M} K d M_{g}=2 \pi \mathcal{X}(M) .
\end{aligned}
$$

Thus, the inequality above must be an equality, which implies $K \equiv 0$ and $\psi \equiv 0$ and, in turn, $\mathcal{X}(M)=0$. This means that $M$ is diffeomorphic to a torus and $\xi$ is parallel. Also, since $\nabla J=0$, we get that $J \xi$ is also parallel. Therefore, $|\xi|=|J \xi|$ are constant on $M$, and since $\xi$ is nontrivial, neither of these vectors does vanish on $M$. 
Since $M$ is diffeomorphic to a torus, a theorem of Cartan assures the existence of closed geodesics $\gamma_{1}$ and $\gamma_{2}$ in $M$, representing the free homotopy classes of a set of generators of $\pi_{1}(M)$. Letting the flow of $\xi$ act (by isometries, since $\xi$ is parallel) on $\gamma_{1}$ and $\gamma_{2}$, we can assume that both of them start and end at the same point $p$ of $M$, so that $\pi_{1}(M ; p)$ is generated by $\left[\gamma_{1}\right]$ and $\left[\gamma_{2}\right]$. If $\gamma_{1}^{\prime}(0)=\alpha \xi+\beta J \xi$, for some $\alpha, \beta>0$, then the parallelism of $\xi$ and $J \xi$ give that $\gamma_{1}^{\prime}=\alpha \xi+\beta J \xi$ along $\gamma_{1}$, so that $\gamma_{1}$ is a geodesic loop based at $p$. Accordingly, so is $\gamma_{2}$ (see Figure 1).
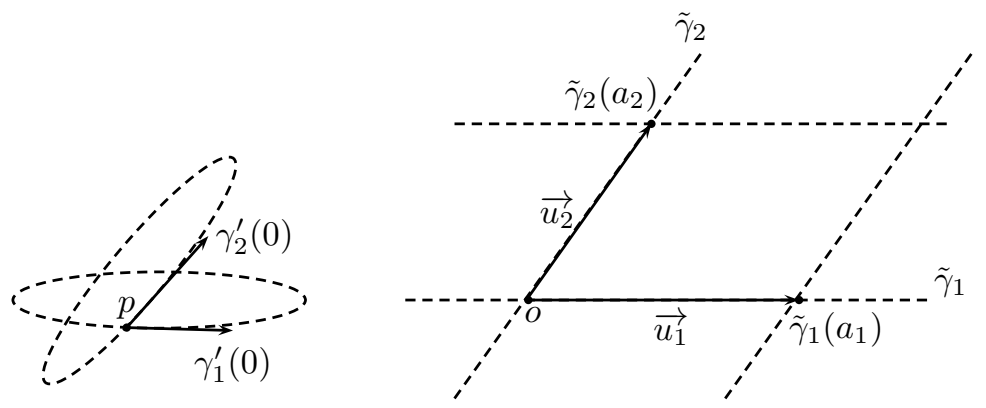

Figure 1. Closed geodesics in $\Sigma$.

Since $K \equiv 0$ and $K$ is diffeomorphic to a torus, its universal covering, endowed with the covering metric, is $\mathbb{R}^{2}$ with its standard flat metric. Let $\pi: \mathbb{R}^{2} \rightarrow M$ be the covering map, $o \in \pi^{-1}(p)$, and $\tilde{\gamma}_{1}$ and $\tilde{\gamma}_{2}$ be the liftings of $\gamma_{1}$ and $\gamma_{1}$ passing through $p$, respectively. Since $\pi$ is a local isometry, $\tilde{\gamma}_{1}$ and $\tilde{\gamma}_{2}$ are straight lines through $o$. Letting $\tilde{\gamma}_{1}(0)=\tilde{\gamma}_{2}(0)=o$, we can assume that $d \pi_{o}$ maps $\tilde{\gamma}_{j}^{\prime}(0)$ to $\gamma_{j}^{\prime}(0)$, for $j=1,2$, as well as that $\angle\left(\tilde{\gamma}_{1}^{\prime}(0), \tilde{\gamma}_{2}^{\prime}(0)\right)=\angle\left(\gamma_{1}^{\prime}(0), \gamma_{2}^{\prime}(0)\right)$ as oriented angles. Suppose further (also without loss of generality) that $\gamma_{1}$ and $\gamma_{2}$ (and so $\tilde{\gamma}_{1}$ and $\tilde{\gamma}_{2}$ ) are normalized, and let $\ell\left(\gamma_{j}\right)=a_{j}$, for $j=1,2$. If $\vec{u}_{1}$ and $\vec{u}_{2}$ are the vectors in $\mathbb{R}^{2}$ that go from $o$ to $\tilde{\gamma}_{1}\left(a_{1}\right)$ and $\tilde{\gamma}_{2}\left(a_{2}\right)$, respectively, and $\mathcal{L}$ is the lattice in $\mathbb{R}^{2}$ generated by $\vec{u}_{1}$ and $\vec{u}_{2}$, then a standard covering argument shows that $M$ is isometric to the flat torus $\mathbb{R}^{2} / \operatorname{Deck}(\pi)$, quotient of the fundamental domain of $\mathcal{L}$ by $\pi$.

Remark 3.3. The nonpositivity of the holomorphic sectional curvature and the compactness of $M$ cannot be relaxed, as shown by the examples of $\mathbb{S}^{2}$ and $\mathbb{R}^{2}$, both of which possess nonparallel closed conformal vector fields.

We now turn to the case of a complete Kählerian manifold $M^{n}$ of complex dimension $n>1$, and first recall (cf. [7, Lemma 1]) that if it has a closed conformal vector field $\xi \in \mathfrak{X}(M) \backslash\{0\}$, then the zeros of $\xi$ are automatically isolated. We first need some auxiliary results.

Lemma 3.4. Let $n>1$ and $\left(M^{n}, g, J\right)$ be a Kählerian manifold endowed with a closed conformal vector field $\xi \in \mathfrak{X}(M) \backslash\{0\}$. Then, the distribution $\mathcal{D}$ generated 
by $\xi$ and $J \xi$ is involutive in $M \backslash \xi^{-1}(0)$, and its leaves are totally geodesic and Kählerian in the induced metric.

Proof. The parallelism of $J$ and the closed conformal character of $\xi$ give $[\xi, J \xi]=$ $\nabla_{\xi} J \xi-\nabla_{J \xi} \xi=J \nabla_{\xi} \xi-\psi J \xi=0$. Moreover, if $\Sigma$ is a leaf of $\mathcal{D}$ and we let $\mathcal{N}$ denote the Nijenhuis tensor of $\Sigma$, it is immediate to see that $\mathcal{N}=0$, so that $\Sigma$ is a complex curve in $\left(M^{n}, J, g\right)$ and the restriction of $g$ to $\Sigma$ (which we shall also call $g$ ) is Kählerian. Now, let $\alpha$ be the second fundamental form of $\Sigma$ and $(\cdot)^{\perp}$ denote orthogonal projection onto $T(\Sigma, g)^{\perp}$. Then, $\alpha(\xi, J \xi)=\left(\nabla_{\xi} J \xi\right)^{\perp}=\left(J \nabla_{\xi} \xi\right)^{\perp}=$ $(\psi J \xi)^{\perp}=0$.

Lemma 3.5. Let $n>1$ and $\left(M^{n}, g, J\right)$ be a connected Kählerian manifold endowed with a closed conformal vector field $\xi \in \mathfrak{X}(M) \backslash\{0\}$. If there exists a nontrivial leaf $\Sigma$ of the distribution $\mathcal{D}$ generated by $\xi$ and $J \xi$ such that the conformal factor of $\xi$ vanishes on $\Sigma$, then it vanishes on $M$. In particular, $\xi$ and $J \xi$ are parallel and do not vanish at any point of $M$.

Proof. By hypothesis, we have $\xi \neq 0$ on $\Sigma$. For $p \in \Sigma$, take a neighborhood $V$ of $p$ in $M \backslash \xi^{-1}(0)$ such that there exists in $V$ a smooth vector field $X$, with $X \perp \xi, J \xi$. Letting $\psi$ stand for the conformal factor of $\xi$ and $\alpha$ be an integral curve of $X$ starting at $q \in V \cap \Sigma$, it follows from (2.4) that

$$
\frac{d}{d t} \psi(\alpha(t))=\left\langle\nabla \psi(\alpha(t)), \alpha^{\prime}(t)\right\rangle=-\langle\operatorname{Ric}(\hat{\xi}) \xi, X\rangle_{\alpha(t)}=0 .
$$

Hence, $\psi$ is constant along $\alpha$ and, since $\psi(q)=0$, we get that $\psi \equiv 0$ along $\alpha$. Since $X$ was arbitrarily chosen subjected to the condition $X \perp \xi, J \xi$, we conclude that $\psi \equiv 0$ in a neighborhood of $p$ in $M \backslash \xi^{-1}(0)$.

The discussion on [5, Section 1] assures that $\psi$ and $\xi$ are uniquely determined by the values of $\psi, \nabla \psi, \xi$, and $\nabla \xi$ at a single point of $M$. Therefore, since $\psi$ vanishes on an open subset of $M \backslash \xi^{-1}(0)$, and (as we have observed above, for $n>1$ ) such a set is connected, we conclude that $\psi \equiv 0$ on $M \backslash \xi^{-1}(0)$. However, since $\xi^{-1}(0)$ is a set of isolated points, we actually have that $\psi \equiv 0$ on $M$. In turn, this shows that both $\xi$ and $J \xi($ since $\nabla J=0)$ are parallel on $M$.

Finally, for $X \in \mathfrak{X}(M)$ we have $X\langle\xi, \xi\rangle=2\langle\psi X, \xi\rangle=0$, so that $|\xi|^{2}$ is constant on $M$. Since $\xi=0$ at most at a set of isolated points, this implies that $|J \xi|=|\xi|$ is constant and positive on $M$.

Lemma 3.6. Let $M$ be a Riemannian manifold, $\eta \in \mathfrak{X}(M) \backslash\{0\}$ be a parallel and complete vector field with flow $\Phi: \mathbb{R} \times M \rightarrow M$, and $\alpha:[0,1] \rightarrow M$ be a geodesic preserved by $\Phi$. If $P_{\alpha}: T_{\alpha(0)} M \rightarrow T_{\alpha(1)} M$ stands for the parallel transport along $\alpha$, then $P_{\alpha}=\left(d \Phi^{1}\right)_{\alpha(0)}$.

Proof. Let $p=\alpha(0)$ and $v \in T_{p} M$. It suffices to show that $t \mapsto\left(d \Phi^{t}\right)_{p}(v)$ is parallel along $\alpha$. To this end, let $\delta:(-\epsilon, \epsilon) \rightarrow M$ be such that $\delta(0)=p$ and $\delta^{\prime}(0)=v$. 
Then,

$$
\begin{aligned}
\frac{D}{d t}\left(d \Phi^{t}\right)_{p}(v) & =\left.\frac{D}{d t} \frac{\partial}{\partial s} \Phi(t, \delta(s))\right|_{s=0}=\left.\frac{D}{d s} \frac{\partial}{\partial t} \Phi(t, \delta(s))\right|_{s=0} \\
& =\left.\frac{D}{d s} \eta(\Phi(t, \delta(s)))\right|_{s=0}=\left(\nabla_{\frac{d}{d s} \Phi(t, \delta(s))} \eta\right)_{\Phi(t, p)}=0
\end{aligned}
$$

since $\eta$ is parallel.

For what follows, we recall that, given a connected submanifold $N$ of a Riemannian manifold $M, p \in N$, and a closed and piecewise differentiable curve $\alpha:[0,1] \rightarrow N$ such that $\alpha(0)=p$, parallel translation along $\alpha$ defines a linear operator $P_{\alpha}: T_{p} N^{\perp} \rightarrow T_{p} N^{\perp}$. More precisely, for $v \in T_{p} N^{\perp}$, we set $P_{\alpha}(v)=V(1)$, where $t \mapsto V(t)$ is the parallel transport of $v$ along $\alpha$. It is immediate to check that the set of such linear operators, endowed with the product $P_{\alpha} \cdot P_{\beta}=P_{\alpha \cdot \beta}$, form a closed subgroup of $O\left(T_{p} N^{\perp}\right)$, called the normal holonomy subgroup of $N$ at $p$ and denoted by $\operatorname{Hol}_{p}^{\perp}(N)$ (for more details, see [2, Chapter 4]). If $q \in N$ and $\delta$ is a piecewise smooth curve in $N$ joining $p$ and $q$, we have $\operatorname{Hol}_{p}^{\perp}(N) \simeq \operatorname{Hol}_{q}^{\perp}(N)$ via $P_{\alpha} \mapsto P_{\delta^{-1} \cdot \alpha \cdot \delta}$. Therefore, from now on we shall refer to the normal holonomy group of $N$, which will henceforth be denoted by $\operatorname{Hol}^{\perp}(N)$.

We then have our second main result.

Theorem 3.7. Let $n>1$ be an integer and $\left(M^{n}, g, J, \nabla\right)$ be a connected, complete Kählerian manifold endowed with a closed, conformal and nontrivial vector field $\xi$. Let $\mathcal{D}$ denote the distribution in $M \backslash \xi^{-1}(0)$ generated by $\xi$ and $J \xi$, and assume that $\mathcal{D}$ has a compact leaf $\Sigma$. If the holomorphic sectional curvature of $M$ along $\Sigma$ is nonpositive and $\mathrm{Hol}^{\perp}(\Sigma)$ is a torsion group, then:

(a) $\xi^{-1}(0)=\emptyset$ and both $\xi$ and $J \xi$ are parallel along $M$.

(b) The leaves of $\mathcal{D}$, endowed with the induced metric, form a family of totally geodesic isometric tori.

(c) The distribution $\mathcal{D}^{\perp}$ is integrable and, in the induced metric, their leaves are totally geodesic, isometric complete Kählerian manifolds of complex dimension $n-1$.

Proof. Lemma 3.4 assures that $\Sigma$ is totally geodesic in $M$, so that its Gaussian curvature $K_{\Sigma}$ coincides with the holomorphic sectional curvature of $M$ along $\Sigma$. Therefore, $K_{\Sigma} \leq 0$, and we can apply Theorem 3.2 to conclude that $\Sigma$ is isometric to a flat torus (hence, $K_{\Sigma} \equiv 0$ ) and the conformal factor $\psi$ of $\xi$ vanishes along it. Lemma 3.5 then shows that $\psi \equiv 0$ on $M, \xi^{-1}(0)=\emptyset$, and $\xi$ and $J \xi$ are parallel along $M$. This establishes (a).

For (b), let $d_{M}$ stand for the Riemannian distance on $M$. Given $q \in M$, the compactness of $\Sigma$ assures the existence of a point $p \in \Sigma$ such that $d_{M}(p, q)=$ $d_{M}(p ; \Sigma)$. Since $M$ is complete, the Hopf-Rinow theorem guarantees the existence of a normalized geodesic $\gamma:[0, l] \rightarrow M$ such that $\gamma(0)=p, \gamma(l)=q$, and $l=$ $\ell(\gamma)=d_{M}(p ; q)$. In particular, it is a well known fact that $\gamma^{\prime}(0) \perp T_{p} \Sigma$, so that 
$\left\langle\gamma^{\prime}(0), \xi_{p}\right\rangle=\left\langle\gamma^{\prime}(0), J \xi_{p}\right\rangle=0$. Now, the parallelism of $\xi$ assures that, along $\gamma$,

$$
\frac{d}{d s}\left\langle\gamma^{\prime}, \xi\right\rangle_{\gamma(s)}=\left\langle\frac{D \gamma^{\prime}}{d s}, \xi\right\rangle_{\gamma(s)}+\left\langle\gamma^{\prime}, \frac{D \xi}{d s}\right\rangle_{\gamma(s)}=0 .
$$

Then, $\left\langle\gamma^{\prime}, \xi\right\rangle$ is constant along $\gamma$, so that $\left\langle\gamma^{\prime}, \xi\right\rangle_{q}=\left\langle\gamma^{\prime}, \xi\right\rangle_{p}=0$. Analogously, $\left\langle\gamma^{\prime}, J \xi\right\rangle_{q}=0$. On the other hand, if $\Sigma_{q}$ is the leaf of $\mathcal{D}$ passing through $q$, then $T_{q} \Sigma_{q}$ is generated by $\xi_{q}$ and $J \xi_{q}$, so that $\gamma^{\prime}(l) \perp T_{q} \Sigma_{q}$ (cf. Figure 2).

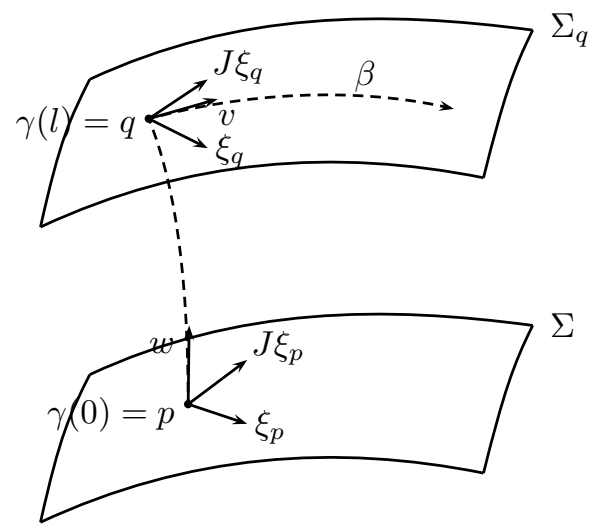

Figure 2. Comparing $\Sigma$ to $\Sigma_{q}$.

By Lemma 3.4, $\Sigma_{q}$ is totally geodesic in $M$. For $v \in T_{q} \Sigma_{q}$, the maximal geodesic of $\Sigma_{q}$ departing from $q$ with velocity $v$ coincides with that of $M$, which is complete. Hence, $\Sigma_{q}$ is also complete. Let $K_{\Sigma_{q}}$ stand for the Gaussian curvature of $\Sigma_{q}$ and $K(\xi, J \xi)$ for the holomorphic sectional curvature of $M$ along the planes generated by $\xi$ and $J \xi$. Letting $R$ denote the curvature operator of $M$, the parallelism of $\xi$ and $J \xi$ give $R(J \xi, \xi) \xi=0$, so that $K(\xi, J \xi) \equiv 0$. However, since $\Sigma_{q}$ is totally geodesic in $M$, we conclude that $K_{\Sigma_{q}}=K(\xi, J \xi)_{\mid \Sigma_{q}}=0$. Therefore, being a connected, complete flat surface, $\Sigma_{q}$ is isometric to a torus, a plane or a cylinder over a plane curve. In what comes next, we shall show that is is isometric to a torus.

The proof of Theorem 3.2 assures the existence of geodesic loops $\alpha_{1}$ and $\alpha_{2}$ in $\Sigma$, based at $p$ and such that $\pi_{1}(\Sigma ; p)$ is generated by $\left[\alpha_{1}\right]$ and $\left[\alpha_{2}\right]$. Let $\alpha=\alpha_{1}$ : $[0,1] \rightarrow \Sigma$ and write $\alpha^{\prime}(0)=v=a \xi_{p}+b J \xi_{p}$, for some $a, b \in \mathbb{R}$. If $v=a \xi_{q}+b J \xi_{q}$, we already know that $v \in T_{q} \Sigma_{q}$. If $\beta$ is the geodesic of $\Sigma_{q}$ departing from $q$ with velocity $v$, we claim that $\beta$ is a geodesic loop, with length $\ell(\beta)=\ell(\alpha)$. We shall prove this in two steps (cf. Figure 3):

(i) Let $w=\gamma^{\prime}(0) \in T_{p}^{\perp} \Sigma$ and $\eta=a \xi+b J \xi$, which is a parallel and (by the completeness of $M$ ) complete vector field in $M$. If $\Phi: \mathbb{R} \times M \rightarrow M$ is the flow of $\eta$, then $\Phi^{t}: M \rightarrow M$ is an isometry, so that $\gamma^{t}(s):=\Phi(t, \gamma(s))$ is a geodesic of $M$ joining $\alpha(t)$ to $\beta(t)$. The parallelism of $\eta$ assures, through Lemma 3.6, that $w(t)=\left(d \Phi^{t}\right)_{p} w$ is parallel along $\alpha$ and normal to $\Sigma$. Hence, $w(1)=P_{\alpha}(w)$, with 


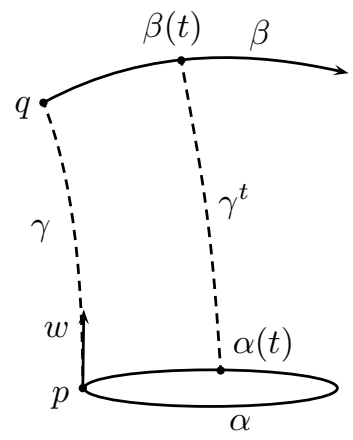

Figure 3. $\beta$ is also a geodesic loop.

$P_{\alpha} \in \operatorname{Hol}^{\perp}(\Sigma)$. Since this is a torsion group by hypothesis, there exists an integer $m \geq 1$ such that $P_{\alpha}^{m}=\mathrm{Id}: T_{p}^{\perp} \Sigma \rightarrow T_{p}^{\perp} \Sigma$; in particular, $P_{\alpha}^{m}(w)=w$. However, since $P_{\alpha}^{m}=P_{\alpha \cdot \ldots \cdot \alpha}(m$ times $)$, if we set $c=\ell(\alpha \cdot \ldots \cdot \alpha)=m \ell(\alpha)$ we conclude that $\gamma^{c}:[0, l] \rightarrow M$ is a geodesic joining $p=(\alpha \cdot \ldots \cdot \alpha)(0)=p$ to $\beta(c)$ and with initial velocity $w$. Then by uniqueness we get $\gamma^{c}=\gamma$, so that $\beta(c)=\gamma^{c}(l)=\gamma(l)=q$. Since $\beta^{\prime}(0)$ is parallel to $\alpha^{\prime}(0)$ along $\gamma, \beta^{\prime}(c)$ is parallel to $\alpha^{\prime}(c)$ along $\gamma^{c}$, and $\alpha^{\prime}(0)=\alpha^{\prime}(c), \gamma=\gamma^{c}$, we conclude that $\beta^{\prime}(0)=\beta^{\prime}(c)$.

(ii) For $0 \leq t \leq l$, let $v_{t} \in \Sigma_{\gamma(t)}$ denote the parallel transport of $v$ along $\gamma_{\mid[0, t]}$ and (according to the discussion in (i)) let $\delta_{t}$ be the geodesic loop that departs from $\gamma(t)$ with initial velocity $v_{t}$, so that $\delta_{0}=\alpha$ and $\delta_{l}=\beta$ (cf. Figure 4). Since exp : $T M \rightarrow M$ is smooth, the function $t \mapsto \ell\left(\delta_{t}\right)$ is continuous; on the other hand (also from (i)), $\frac{\ell\left(\delta_{t}\right)}{\ell(\alpha)} \in \mathbb{Z}$ for each $t \in[0, l]$. In particular, $\ell(\beta)=\ell\left(\delta_{l}\right)=\ell\left(\delta_{0}\right)=\ell(\alpha)$.

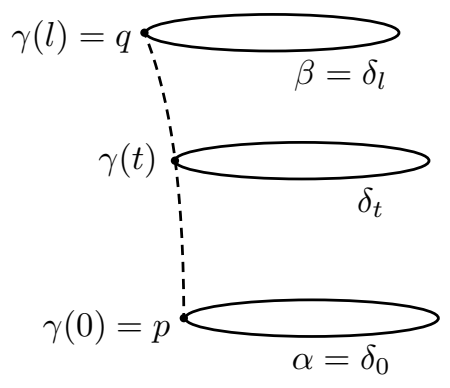

Figure 4. Computing the length of $\beta$.

The argument in (i) guarantees that the geodesics $\beta_{1}$ and $\beta_{2}$ of $\Sigma_{q}$, obtained from $\alpha_{1}$ and $\alpha_{2}$ by parallel transporting $\alpha_{1}^{\prime}(0)$ and $\alpha_{2}^{\prime}(0)$ along $\gamma$, are distinct geodesic 
loops in $\Sigma_{q}$. Since a plane has no closed geodesics and a cylinder over a plane curve has only one geodesic loop through each one of its points, we conclude that $\Sigma_{q}$ is a torus. Also, it follows by construction that $\left\langle\alpha_{1}^{\prime}(0), \alpha_{2}^{\prime}(0)\right\rangle=\left\langle\beta_{1}^{\prime}(0), \beta_{2}^{\prime}(0)\right\rangle$, and (ii) assures that $\ell\left(\alpha_{1}\right)=\ell\left(\beta_{1}\right)$ and $\ell\left(\alpha_{2}\right)=\ell\left(\beta_{2}\right)$. Therefore, the last part of the proof of Theorem 3.2 assures that $\Sigma_{q}$ is isometric to $\Sigma$.

We now turn to (c). If $X$ and $Y$ are smooth vector fields in $\mathcal{D}^{\perp}$, then the parallelism of $\xi$ and $J \xi$ give $\left\langle\nabla_{X} Y, \xi\right\rangle=0$ and $\left\langle\nabla_{X} Y, J \xi\right\rangle=0$. In particular, $[X, Y] \in \mathcal{D}^{\perp}$, so that $\mathcal{D}^{\perp}$ is integrable. Letting $N$ denote a leaf of $\mathcal{D}^{\perp}$ and $\alpha$ its second fundamental form, we have

$$
\alpha(X, Y)=\left\langle\nabla_{X} Y, \xi\right\rangle \frac{\xi}{|\xi|^{2}}+\left\langle\nabla_{X} Y, J \xi\right\rangle \frac{J \xi}{|\xi|^{2}}=0
$$

and $N$ is totally geodesic in $M$. The completeness of $N$ (in the induced metric) now follows from that of $M$, together with the fact that geodesics in $N$ are also geodesics in $M$.

It is immediate to check that $X \in \mathfrak{X}(N) \Rightarrow J X \in \mathfrak{X}(N)$. Therefore, $J$ is an almost complex structure on $N$, and the fact that the Levi-Civita connection of $N$ is the restriction of that of $M$ guarantees that $J$ is parallel on $N$. Finally, since the Nijenhuis tensor of $N$ is the restriction of that of $M$, which vanishes identically, we conclude that $N$ is a Kählerian manifold in the induced metric.

For the last part, we argue pretty much as in (i). To this end, let $N_{1}$ and $N_{2}$ be two distinct leaves of $\mathcal{D}^{\perp}$, and take $p_{1} \in N_{1} \cap \Sigma$ and $p_{2} \in N_{2} \cap \Sigma$. Let $\delta:[0, a] \rightarrow \Sigma$ be a geodesic of $\Sigma$ joining $p_{1}$ to $p_{2}$, and $\delta^{\prime}(0)=a \xi_{p_{1}}+b J \xi_{p_{1}}$ for some $a, b \in \mathbb{R}$. The parallelism of $\xi$ and $J \xi$ assure that $\delta^{\prime}$ is the restriction of the parallel (hence, complete) vector field $\eta=a \xi+b J \xi$ to $\delta$. If $\Phi: \mathbb{R} \times M \rightarrow M$ denotes the flow of $\eta$, then $\Phi^{a}: M \rightarrow M$ is an isometry such that $\Phi^{a}\left(p_{1}\right)=p_{2}$ and $\left(d \Phi^{a}\right)_{p_{1}}\left(T_{p_{1}} N_{1}\right)=T_{p_{2}} N_{2}$. Since $N_{1}$ and $N_{2}$ are connected, complete and totally geodesic in $M$, an argument pretty much like the one presented in the proof of (b) guarantees that $\Phi^{a}$ applies geodesics in $N_{1}$ to geodesics in $N_{2}$. Hence, $\Phi^{a}\left(N_{1}\right) \subset N_{2}$ and, likewise, $\Phi^{-a}\left(N_{2}\right) \subset N_{1}$. Thus, $\Phi^{a}\left(N_{1}\right)=N_{2}$.

Corollary 3.8. Let $n>1$ be an integer and $\left(M^{n}, g, J, \nabla\right)$ be a connected, complete Kählerian manifold satisfying the hypotheses of the previous result. If $\tilde{M}$ stands for the universal covering of $M$, endowed with the covering metric, then $\tilde{M}$ is isometric to a Riemannian product $\tilde{N} \times \mathbb{R}^{2}$, where $\tilde{N}$ is a connected, simply connected, complete Kählerian manifold.

Proof. Letting $\tilde{g}$ denote the covering metric, the covering map $\pi: \tilde{M} \rightarrow M$ turns into a local isometry, so that $\tilde{M}$ is naturally a Kählerian manifold. Moreover, the orthogonal foliations on $M$ lift to two orthogonal foliations of $\tilde{M}$ with totally geodesic leaves, and one of which has leaves isometric to $\mathbb{R}^{2}$. Since $\tilde{M}$ is simply connected, it now suffices to apply the complex version of the De Rham decomposition theorem. 


\section{ACKNOWLEDGMENTS}

The author wishes to thank professor Jorge V. Pereira for some helpful conversations.

\section{REFERENCES}

[1] L. J. Alías, D. Impera and M. Rigoli. Spacelike hypersurfaces of constant higher order mean curvature in generalized Robertson-Walker spacetimes. Math. Proc. Cambridge Philos. Soc. 152 (2012), no. 2, 365-383. MR 2887879

[2] J. Berndt, S. Console and C. Olmos. Submanifolds and Holonomy. Chapman \& Hall/CRC, Boca Raton, 2003. MR 1990032

[3] H. F. de Lima and U. L. Parente. On the geometry of maximal spacelike hypersurfaces immersed in a generalized Robertson-Walker spacetime. Ann. Mat. Pura Appl. (4) 192 (2013), no. 4, 649-663. MR 3081639

[4] S. Montiel. Uniqueness of spacelike hypersurfaces of constant mean curvature in foliated spacetimes. Math. Ann. 314 (1999), no. 3, 529-553. MR 1704548

[5] M. Obata. Conformal transformations of Riemannian manifolds. J. Diff. Geom. 4 (1970), 311-333. MR 0267485

[6] G. Pitiş. Integral submanifolds with closed conformal vector field in Sasakian manifolds. New York J. Math. 11 (2005), 157-170. MR 2154352

[7] A. Ros and F. Urbano. Lagrangian submanifolds of $\mathbb{C}^{n}$ with conformal Maslov form and the Whitney sphere. J. Math. Soc. Japan 50 (1998), no. 1, 203-226. MR 1484619

[8] S. Tanno and W. C. Weber. Closed conformal vector fields. J. Diff. Geom. 3 (1969) 361-366. MR 0261498

\section{A. Caminha}

Departamento de Matemática, Universidade Federal do Ceará, 60440-554 Fortaleza, CE, Brazil caminha@mat.ufc.br

Received: August 14, 2018

Accepted: March 7, 2019 